\title{
ON FIXED POINTS OF SET-VALUED DIRECTIONAL CONTRACTIONS
}

\author{
SEHIE PARK \\ Department of Mathematics \\ Seoul National University \\ Seoul 151, KORE.A \\ (Received June 4, 1984 and in revised form April 25, 1985)
}

ABSTRACT. Using equivalent formulations of Ekeland's theorem, we improve fixed point theorems of Clarke, Sehgal, Sehgal-Smithson, and Kirk-Ray on directional contractions by giving geometric estimations of fixed points.

KIYY WORDS AIID PHRASES. Z.s.c. function, (weak) directional contraction, fixed point, :iutionary roint, Hansdorff psuedomitric.

1.18: AMS SUBJECI CLASSIFICATION CODE'S. 47H10, 54H25.

\section{INTRODUCTION ANI) PRELININARIES}

In $|1|,|2|$, Sehgal and Smithson proved fixed point theorems for set-valued weak directional contractions which extend earlier results of Clarke [3], Kirk and Ray [4], and Assad and Kirk [5]. In the present paper, results in [1], [2] are substantially strengthened by giving geometric estimations of locations of fixed points.

The following equivalent formulations $\{6\rfloor$ of the well-known central result of Ekeland [7|, |8] on the variational principle for approximate solutions of minimization problems is used in the proofs of the main results.

THEOREM 1. Let $(\mathrm{V}, \mathrm{d})$ be a complete metric space, and $\phi: \mathrm{V} \rightarrow \mathrm{R} \cup\{+\infty\}$ a l.s.c. function, $t+\infty$, bounded from below. Let $\varepsilon>0$ and $\lambda>0$ be given, and a point $u \in V$ such that

$$
F(u) \leq \inf _{V} F+\varepsilon \text {. }
$$

Let $S(\lambda)=\left\{x \in V \mid F(x) \leq F(u)-\varepsilon \lambda^{-1} d(u, x)\right\}$. Then the following equivalent conditions hold:

(i) There exists a point $v \varepsilon S(\lambda)$ satisfying

$$
F(w)>F(v)-\varepsilon \lambda^{-1} d(v, w) \text { for } \forall w \neq v \text {. }
$$

(ii) If $T: S(\lambda) \rightarrow 2^{V}$ is a set-valued map satisfying the condition

$$
\begin{aligned}
\forall \mathrm{x} \varepsilon S(\lambda) \backslash \mathrm{T}(\mathrm{x}) & \exists \mathrm{y} \varepsilon \mathrm{V} \backslash\{\mathrm{x}\} \text { such that } \\
& \mathrm{F}(\mathrm{y}) \leq \mathrm{F}(\mathrm{x})-\varepsilon \lambda^{-1} \mathrm{~d}(\mathrm{x}, \mathrm{y}),
\end{aligned}
$$

then $T$ has a fixed point $v \& S(\lambda)$.

(iii) If $f: S(\lambda) \rightarrow V$ satisfies

$F(f x) \leq F(x)-\varepsilon \lambda^{-1} d(x, f x)$

for all $x \in S(\lambda)$, then $f$ has a fixed point $v \in S(\lambda)$.

In Theorem $1,2^{\mathrm{V}}$ denotes the power set of $\mathrm{V}$. Note that 
$S(\lambda) \subset\{x \in V \mid F(x) \leq F(u), d(u, x) \leq \lambda\} \subset \bar{B}(u, \lambda)$

and $\mathrm{fS}(\lambda) \subset \mathrm{S}(\lambda)$, where $\bar{B}$ denotes the closed ball.

Throughout this paper, $(V, d)$ denotes a metric space and $B(V)$ denotes the class of all nonempty bounded subsets of $V$ with the Hausdorff pseudometric $H$ defined by

$$
H(A, B)=\max \left\{\sup _{x \in A} d(x, B), \sup _{y \in B} d(y, A)\right\} \text {. }
$$

Also, $C(V)$ denotes the class of all nonempty compact subsets of $V$. For an $x \in V$ and $\mathrm{A} \in \mathrm{C}(\mathrm{V})$, we put

$$
[x, A]=\{y \in A \mid d(x, y)=d(x, A)\},
$$

which is nonempty. For $x, y \in V$, we denote

$$
[x, y]=\{z \in \vee \mid d(x, z)+d(z, y)=d(x, y)\},
$$

and

$$
(x, y]=\lfloor x, y\} \backslash\{x\},(x, y)=(x, y] \backslash\{y\} .
$$

Let $S$ be a nonempty subset of $V$ and $T: S \rightarrow C(V)$ be a set-valued map. For $x \in S$ and $A \in C(V)$, the weak directional derivative DT $(x, y)$ of $T$ at $x$ in the direction of a $y \in[x, T(x)]$ is defined by

$$
\operatorname{DT}(x, y)= \begin{cases}0, & \text { if } x=y, \\ \inf \left\{\frac{H(T x, T z)}{d(x, z)} \mid z \varepsilon(x, y] \cap S\right\}, & \text { if }(x, y] \cap S \neq \phi, \\ \infty, & \text { if }(x, y] \cap S=\phi .\end{cases}
$$

$A$ map $T: S \rightarrow C(V)$ is called a weak directional contraction if there exists a $k \varepsilon[0,1)$ such that for each $x \in S$, there exists a $y \varepsilon[x, T(x)]$ with $\operatorname{DT}(x, y)$ $<k[2]$.

$\Lambda$ map $T: S \rightarrow B(V)$ is called a directional contraction if there exists a $\mathrm{k} \varepsilon[0,1)$ such that for each $\mathrm{x} \in \mathrm{S}$ and $\mathrm{y} \varepsilon \mathrm{T}(\mathrm{x})$,

$$
H(T(z), T(x)) \leq k d(z, x)
$$

for all $z \in[x, y] \cap S[7]$.

2. RESULTS.

THEOREM 2. Let $S$ be a complete subset of $V$ and $T: S \rightarrow C(V)$ a weak directional contraction for which the function $F(x)=d(x, T(x)), x \in S$, is 1.s.c. Then for any $u \in S$ and $\varepsilon>0$ satisfying $F(u) \leq(1-k) \varepsilon, T$ has a fixed point in $s(\varepsilon) \subset \bar{B}(u, \varepsilon) \cap S$.

PROOF. Choose a point $u \in S$ satisfying $F(u) \leq \inf _{S} F+(1-k) \varepsilon$. Suppose $x \in S(\varepsilon) \backslash T(x)$. Since $T$ is a weak directional contraction, there exists a $y \varepsilon$ $\lfloor x, T(x)\rfloor, \quad x \neq y$, with $\operatorname{DT}(x, y)<k$. Hence, there exists a $z \varepsilon(x, y\rfloor \cap S$ such that

$$
H(T(x), T(z))<k d(x, z) .
$$

Since

$$
d(x, z)+d(z, T(x)) \leq d(x, y)=d(x, T(x)),
$$

we have

$$
\begin{aligned}
d(z, T(z)) & \leq d(z, T(x))+H(T(x), T(z)) \\
& \leq d(x, T(x))-d(x, z)+k d(x, z) \\
& \leq d(x, T(x))-(1-k) d(x, z) .
\end{aligned}
$$


Hence, $F(z) \leq F(x)-(1-k) d(x$, , ) . lherefore, by Therem l(ii i), $T$ has a fixed point $\mathrm{v} \varepsilon \mathrm{S}(\varepsilon)$.

Theorem 2 is an improved version of Theorem (a) of [2] with much simpler proof. In fact, for suitable values of $\varepsilon$ and $k$, the conclusion gives geometric estimations of locations of fixed points. However, for Theorem (b) of [2], such estimation seems to be hard to get.

Note also that for Theorem 1 of Clarke [3], we can apply our Theorem 2 .

The following improves Corollary of [2] and a result of Kirk and Ray [4].

COROLLARY 1. Let $S$ be a closed convex subset of a Banach space $X$ and $T$ : $S, C(S)$ a map for which the function $F(x)=d(x, T(x)), x \varepsilon S$, is 1.s.c. Suppose there exists a $k \in(0,1)$ such that for each $x \in S$ there correspond $a y=y(x)$ $\varepsilon|x, T(x)|$ and a $\delta \varepsilon(0,1)$ satisfying $H(T(x), T(x+\delta(y-x)) \leq k \delta|| y-x||$.

Then the conclusion of Theorem 1 follows.

PROOF. $\Lambda \mathrm{s}$ in the proof in $[2], \mathrm{T}$ is a weak directional contraction with the conatant $\mathrm{k}$.

THEOREM 3. Let $\mathrm{S}$ be a closed subset of a complete metric space $\mathrm{V}$ and $\mathrm{T}$ : $S \rightarrow B(V)$ a directional contraction with the constant $\alpha$. If $T$ satisfies

(a) for each $x \in S$, y $\varepsilon T(x) \backslash S$, there exists a $z \varepsilon(x, y) \backslash S$ with $T(z) \subset S$, and

(b) $\mathrm{g}(\mathrm{x})=\mathrm{d}(\mathrm{x}, \mathrm{T}(\mathrm{x}))$ is l.s.c., then, for any $u \in S, \varepsilon>0$ and $\beta, \alpha<\beta<1$ satisfying $g(u) \leq(1-\beta) \varepsilon$, there exists a fixed point $v$ of $T$ in $S(\varepsilon) \cap S$.

LEMMA [4]. Under the hypothesis of Theorem 3, there exists a map $A: S \rightarrow B(X)$ with the following properties

i) for each $\mathrm{x} \in \mathrm{S}, \mathrm{A}(\mathrm{x}) \neq \phi$ and $\mathrm{A}(\mathrm{x}) \subset \mathrm{T}(\mathrm{x})$

ii) if $y \in A(x)$, then $d(x, y) \leq(1-\beta+\alpha)^{-1} d(x, T(x))$,

iii) if $A(x) \cap S=\phi$ for some $x \in S$, then there exists a $y=y(x) \varepsilon A(x)$ and a $z=z(x, y) \varepsilon(x, y) \cap S$ such that

$$
d(x, y) \leq d(x, T(x))+(\beta-\alpha) d(x, z) .
$$

PROOF OF THEOREM 3. Define a map $f: S \rightarrow S$ as follows: for $x \in S$ such that $A(x) \cap S \neq \phi$, let $f(x)$ be any element of $A(x) \cap S$; and for $x \in S$ such that $A(x) \cap S=\phi$, since there exist $y=y(x) \varepsilon A(x)$ and $z=z(x, y) \varepsilon(x, y) \quad S$ satisfying (2.1) by Lemma, let $f(x)=z$. We claim that for any $x \in S$,

$$
H(T(x), T(f(x))) \leq \alpha d(x, f(x)) .
$$

This is clear if $\Lambda(x) \cap S=\phi$. If $A(x) \cap S \neq \phi$, since $f(x) \varepsilon T(x)$ and $f(x) \varepsilon$ $[x, f(x)] \cap S$, the definition of $T$ implies $(2.2)$. Set $F(x)=(1-B)^{-1} g(x)$. We know that for any $x \in S$ and $y=f(x)$,

$$
F(y) \leq F(x)-d(x, y)
$$

holds as in the proof of [1, Theorem 1]. Therefore, by Theorem 1 (iii), for any $u \varepsilon S$ and $\varepsilon>0$ satisfying $F(u) \leq$ inf $_{S} F+\varepsilon$, there exists a fixed point $v$ of $f$ in $S(\varepsilon) \cap S$. This implies that $v \varepsilon T(v)$ for otherwise $f(v) \notin A(v) \cap S$ and hence by the definition of $f, A(v) \cap S=\phi$. Thus, $f(v) \varepsilon(v, y(v))$ for some $y(v) \varepsilon A(v)$. This contradicts $v \neq f(v)$. Consequently, $v \in T(v)$. Since inf $F=0$, $u$ can be 
chosen so that $F(u) \leq \varepsilon$, that is, $d(u, T(u)) \leq(1-\beta) \varepsilon$. This completes our proof.

Note that Theorem 3 is a strengthened form of [1, Theorem 1].

A metric space is said to be convex if for each $x, y \varepsilon x, x \neq y$, there exists a $z \varepsilon(x, y)$. It is known that if $S$ is a closed subset of a complete convex metric space $V$ and $x \in S$ and $y \notin S$, then there exists a $z \varepsilon[x, y) \cap \delta S$ where $\delta$ is the boundary.

Now, we obtain the following improved version of [1, Corollary 1] as an immediate consequence of Theorem 3.

COROLLARY 2. Let $S$ be a closed subset of a complete convex metric space $V$. Let $T: S \rightarrow B(V)$ be a directional contraction which the constant $\alpha$ such that $T(S) \subset S$. If $g(x)=d(x, T(x))$ is 1.s.c. on $S$, then for any $u \in S, \varepsilon>0$, and $\beta, \quad \alpha<\beta<1$, satisfying $g(u) \leq(1-\beta) \varepsilon$, there exists a fixed point $v$ of $T$ in $S(\varepsilon) \cap S$.

Also, the following improves [1, Corollary 2] and an earlier result of Assad-Kirk $|5|$.

COROLLARY 3. Let $S$ be a closed subset of a complete convex metric space $V$. Suppose $T: S \rightarrow B(X)$ is a contraction, that is, there exists an $\alpha \varepsilon(0,1)$ such that for all $x, y \in S$,

$$
H(T(x), T(y)) \leq \alpha d(x, y) .
$$

If $T(\delta S) \subset S$, then for any $u \varepsilon S, \varepsilon>0$, and $\beta, \alpha<\beta<1$, satisfying

$d(u, T(u)) \leq(1-\beta) \varepsilon$, either $u$ is a fixed point of $T$ or there exists a fixed point $v$ of $T$ in

$$
S(\varepsilon) \cap S \backslash B(u, s)
$$

where $\mathrm{s}=\mathrm{d}(\mathrm{u}, \mathrm{T}(\mathrm{u}))(\mathrm{j}+\alpha)^{-1}$.

PROOF. Since a contraction is a directional contraction and $g(x)=d(x, T(x))$ is continuous, by Corollary 2, $T$ has a fixed point $v \varepsilon S(\varepsilon) \cap S$. Suppose $u$ is not fixed under $T$. Then for any $y \in B(u, s) \cap S$ we have

$$
\begin{aligned}
d(u, T(u)) & \leq d(u, y)+d(y, T(u)) \\
& <s+d(y, T(u)),
\end{aligned}
$$

that is,

$$
\alpha(1+\alpha)^{-1} d(u, T(u))<d(y, T(u))
$$

Hence,

$$
d(y, T(u))>\alpha s>\alpha d(y, u) .
$$

Suppose y $\varepsilon \mathrm{T}(\mathrm{y})$. Then we have

$$
H(T(y), T(u))>\operatorname{ad}(y, u),
$$

a contradiction. This completes our proof.

ACKNOWLEDGEMENT: The author expresses his gratitude to the referee for his kind comments.

This work was partially supported by a grant from the Korea Science and Engineering Foundation, 1983-84. 


\section{REFERENCES}

1. SEHGAL, V. M. Sume fixed point theorems for set valued directional contraction mappings, Internat. J. Math. \& Math. Sci. $\underline{3}$ (1980), 455-460.

2. SEHGAL, V. M. and SMITHSON, R. E. A fixed point theorem for weak directional contraction multifunctions, Math. Japonica 25 (1980), 345-348.

3. ClARKE, F. H. Pointwise contraction criteria for the existence of $f$ ixed points, Canad. Math. Bul1. 21(1978), 7-11.

4. KIRK, W. A. and KAY, W. O. A remark on directional contractions, Proc. Mmer. Math. Soc. $\underline{66}(1977), 279-283$.

5. ASSAD, N. A. and KIRK, K. A. Fixed point theorems for set valued mappings, Pacif ic J. Math. 出(1972), 553-561.

6. PARK, S. Equivalent formulations of Ekeland's variational principle for approximate solutions of minimization problems and their applications, The MSRIKorea Pub1. 1 (1985), to appear.

7. EKELAND, I. On the variatiomal principle, J. Math. Anal. Appl. 47(1974), $324-353$.

8. EKELAND, I. Nonconvex minimization problems, Bu1l. Amer. Math. Soc., 1 (1979), 443-474. 


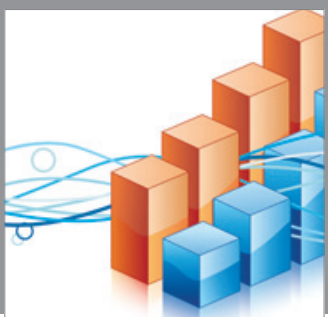

Advances in

Operations Research

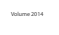

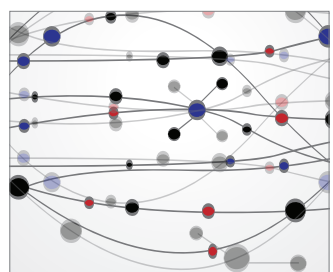

\section{The Scientific} World Journal
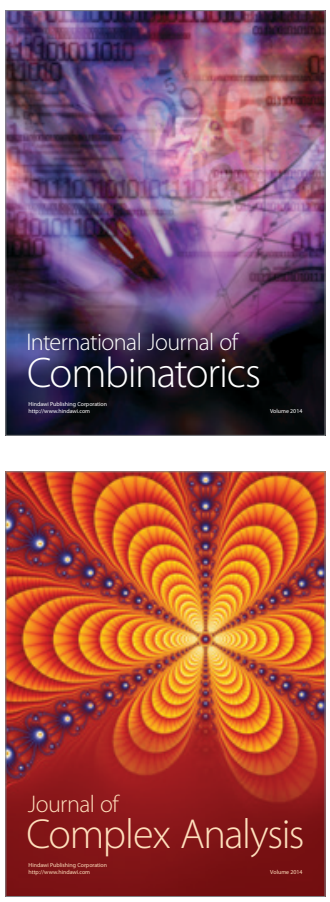

International Journal of

Mathematics and

Mathematical

Sciences
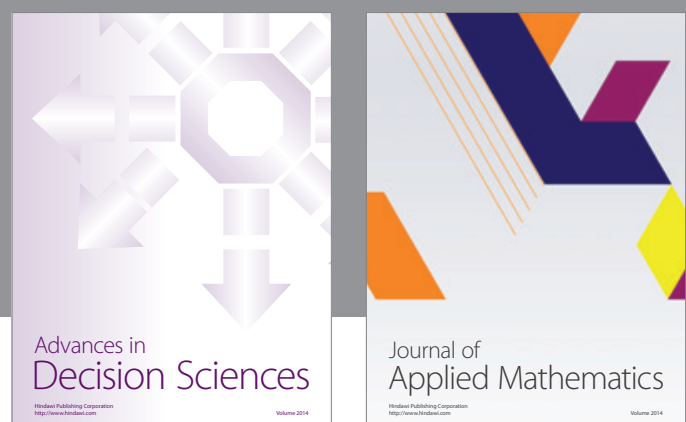

Journal of

Applied Mathematics
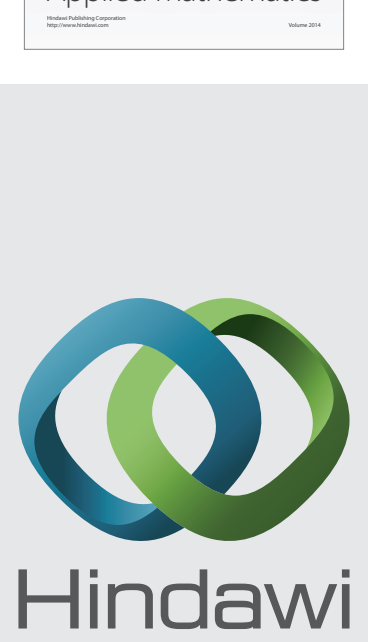

Submit your manuscripts at http://www.hindawi.com
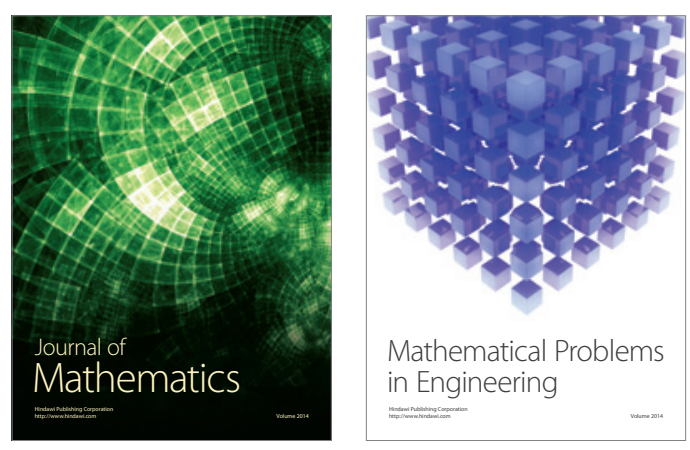

Mathematical Problems in Engineering
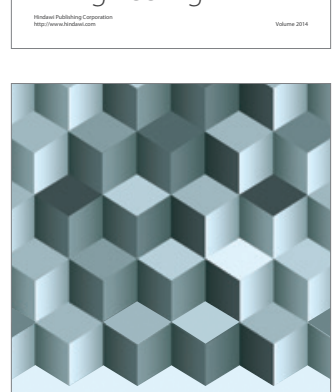

Journal of

Function Spaces
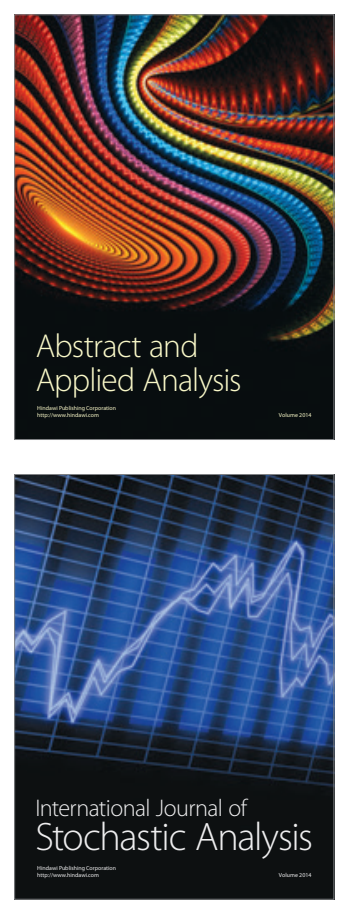

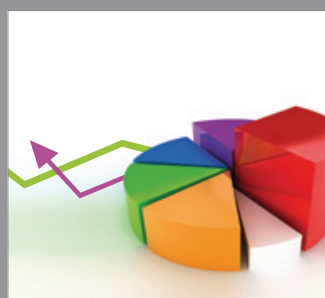

ournal of

Probability and Statistics

Promensencen
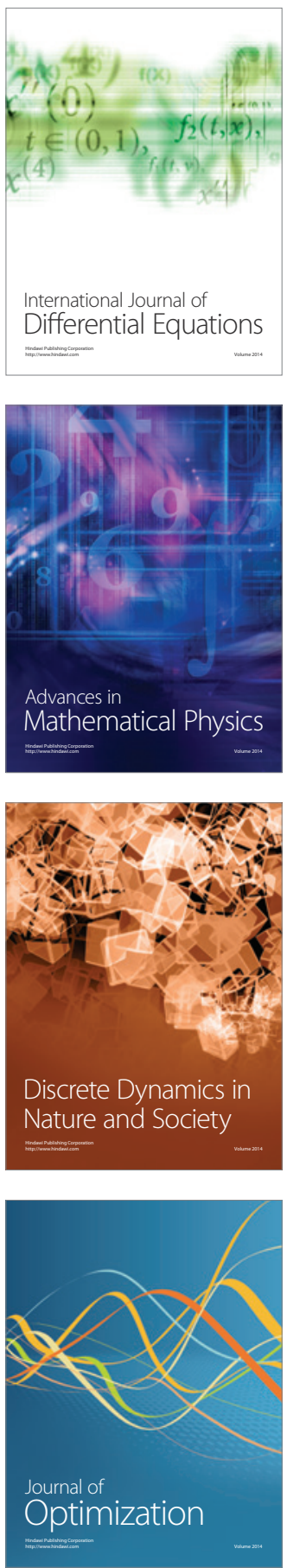